\title{
Effective Lagrangian of the two Higgs doublet model
}

\author{
P. Ciafaloni \\ IFAE, Universitat Autònoma de Barcelona, Edifici Cn, E-08193 Bellaterra, Spain \\ D. Espriu \\ DECM and IFAE, Universitat de Barcelona, Diagonal 647, E-08028 Barcelona, Spain
}

(Received 26 December 1996)

\begin{abstract}
We consider the two Higgs doublet model extension of the standard model in the limit where all physical scalar particles are very heavy, too heavy, in fact, to be experimentally produced in forthcoming experiments. The symmetry-breaking sector can thus be described by an effective chiral Lagrangian. We obtain the values of the coefficients of the $O\left(p^{4}\right)$ operators relevant to the oblique corrections and investigate to what extent some nondecoupling effects may remain at low energies. A comparison with recent CERN LEP data shows that this model is indistinguishable from the standard model with one doublet and with a heavy Higgs boson, unless the scalar mass splittings are large. [S0556-2821(97)04013-7]
\end{abstract}

PACS number(s): 14.80.Cp, 12.39.Fe

\section{INTRODUCTION}

The two Higgs doublet model (2HDM [1]) is one of the most popular extensions of the standard model (SM). It provides a natural way of introducing an additional U(1) (or Peccei-Quinn [2]) symmetry, allows for spontaneous breaking of $C P$ invariance [3], and may provide an interesting phenomenology of flavor-changing neutral currents [4], compatible with the current experimental limits, if the appropriate form of the scalar potential is chosen. In fact if nature has decided that electroweak symmetry breaking should proceed via elementary scalar fields, it is difficult to answer the question as to why not two doublets instead of just one as in the minimal standard model (MSM).

Recent data from the CERN $e^{+} e^{-}$collider LEP [5] put stringent limits on the symmetry-breaking sector of the SM. While composite and QCD-like technicolor models are not, strictly speaking, ruled out yet, they are severely constrained. Technicolor groups larger than $\mathrm{SU}(2)_{\mathrm{TC}}$ appear extremely unlikely, while the amount of custodial symmetry breaking in the techniquark mass sector is severely limited by the $\rho$ parameter [6]. Nature seems to be telling us that whatever physics one may think of adding to the SM, it should, to a good extent, decouple at low energies (in the technical sense of Appelquist and Carazzone [7]). Technicolor and similar theories are nondecoupling; finite and calculable corrections to the low-energy parameters $S, T, U$ [8] or, equivalently, $\varepsilon_{1}, \varepsilon_{2}, \varepsilon_{3}$ [9] remain even when the mass scale of all new particles is large. This is why one is able to set severe limits on such theories. On the contrary, the symmetry-breaking sector of the minimal supersymmetric standard model (MSSM) [10], which contains two Higgs doublets, is decoupling. In such theories one can always choose the parameters in such a way that the additional contribution of this sector to $S, T$, and $U$ is arbitrarily small (at least in the MSSM, see [11]). The fact that the theory is decoupling implies that modifications to the SM results are small and adjustable.

One may wonder whether the fact that the enlarged symmetry-breaking sector decouples from low-energy $\left(\sim M_{W}\right)$ phenomenology is a generic feature of $2 \mathrm{HDM}$ or it is just limited to the MSSM. Does the heavy scalar sector decouple from low-energy $\left(\sim M_{W}\right)$ phenomenology? And if not, to what extent? We propose to investigate this issue here.

Heavy scalar masses usually imply, at least naively, a strongly interacting symmetry-breaking sector thus rendering the usual linear, perturbative approach questionable. We shall thus phrase our discussion in the language of effective Lagrangians. This technique is the natural one when all the physical degrees of freedom in the symmetry-breaking sector are heavy, and a separation between light and heavy degrees of freedom is clear (which is not the same as saying that the heavy sector should necessarily decouple, as exemplified by technicolor models). It should be said right away that the above situation does not correspond to the MSSM, where it is not natural to have all scalar fields heavy, and some light scalar must necessarily be present. Thus our results are not directly applicable to the MSSM. Rather, our analysis applies to 2HDM where the masses of all physical scalar particles are very large, typically somewhere in the $\mathrm{TeV}$ region. While 2HDM models with light to moderate masses have already been studied [12], heavy doublets do not appear to have been considered in detail, at least to our knowledge. The symmetry-breaking sector just described may or (most likely) may not correspond to some supersymmetric theory; this does not concern us here. If additional light fields (such as $s$ quarks or gauginos) are present we shall just include them explicitly in our low-energy theory.

We want to keep the light degrees of freedom only, namely, the gauge and Goldstone bosons. The latter are collected in a unitary matrix $U=\exp \left(2 i G^{a} T^{a} / v\right)$ where $v$ is the vacuum expectation value that gives the $W$ and $Z$ bosons a mass and $G_{a}$ are the Goldstone modes. The matrix $U$ is an element of the $\mathrm{SU}(2) \times \mathrm{SU}(2) / \mathrm{SU}(2)$ coset space. Given this basic building block and gauge invariance one just constructs the most general Lagrangian compatible with the desired symmetries via a derivative expansion: namely $[13,14]$,

$$
\mathcal{L}=\mathcal{L}^{2}+\mathcal{L}^{4}+\cdots
$$


The indices denote the dimensionality of the corresponding operators, i.e., two derivatives, four derivatives, etc. Gauge fields count as one derivative and explicit breaking terms are forbidden on account of gauge invariance. See [13] for a classification of all possible operators up to $O\left(p^{6}\right)$. The information on physics beyond the MSM is encoded in the coefficients of the above effective chiral Lagrangian (ECL).

There are only two independent $O\left(p^{2}\right)$ operators:

$$
\mathcal{L}^{2}=\frac{v^{2}}{4} \operatorname{Tr}\left(D_{\mu} U D^{\mu} U^{\dagger}\right)+a_{0} \frac{v^{2}}{4}\left[\operatorname{Tr}\left(\tau_{3} U^{\dagger} D_{\mu} U\right)\right]^{2} .
$$

The first one is universal, its coefficient is fixed by the $W$ mass. The other one is related to the $\rho$ parameter. In addition, there are a few $O\left(p^{4}\right)$ operators with their corresponding coefficients:

$$
\begin{aligned}
\mathcal{L}^{4}= & \frac{1}{2} a_{1} g g^{\prime} \operatorname{Tr}\left(U B_{\mu \nu} U^{\dagger} W^{\mu \nu}\right) \\
& -\frac{1}{4} a_{8} g^{2} \operatorname{Tr}\left(U \tau^{3} U^{\dagger} W_{\mu \nu}\right) \operatorname{Tr}\left(U \tau^{3} U^{\dagger} W^{\mu \nu}\right)+\cdots
\end{aligned}
$$

In the above expression $B_{\mu \nu}$ and $W_{\mu \nu}$ are the field strength tensors associated with the $\mathrm{SU}(2)$ and $\mathrm{U}(1)$ gauge fields. In this paper we shall only consider the self-energy, or oblique corrections, which are dominant in the two Higgs doublet model just as they are in the MSM. Accordingly, we shall determine only those coefficients of the ECL that contribute to two-point functions to leading and next-to-leading order in the momentum expansion. These consist in just the two operators quoted above after using the equations of motion (see, however, [15]).

Apart from vacuum polarization effects, the 2HDM introduces, with respect to the SM, some additional vertex and box corrections due to the exchange of scalar particles. Let us phrase the discussion in terms of the familiar $\varepsilon_{1}, \varepsilon_{2}, \varepsilon_{3}$ parameters [9]. The experimental bounds on these quantities are extracted from observables with leptons in the external legs and, therefore, such box and vertex corrections are, roughly speaking, suppressed by a factor $\sim\left(m / M_{W} \cos \beta\right)^{2}$ with respect to typical gauge corrections. ${ }^{1}$ Here $m$ is the lepton mass and $\tan \beta=v_{2} / v_{1}$ depends on the ratio between the two vacuum expectation values (VEV's) appearing in 2HDM. With the current experimental limit $\tan \beta$ $<0.52 M_{+}(\mathrm{GeV})$ [17] $\left(M_{+}\right.$is the charged Higgs boson mass), and setting for $M_{+}$the value $M_{+} \approx 600 \mathrm{GeV}$, we get $\tan \beta \leq 300$. Then the additional box and vertex contributions due to scalar exchange can be safely neglected even for the $\tau$ lepton. The limit of vacuum polarization dominance is, therefore, justified and we can compare our results with the values for $\varepsilon_{i}$ extracted from lepton data.

\footnotetext{
${ }^{1}$ This holds for the so-called type II models. For type I models the relevant parameter is $\cot \theta$ instead of $\tan \beta$; apart from this, the same considerations hold. See [16].
}

\section{THE MODEL AND ITS NONLINEAR REALIZATION}

From the considerations in the previous section it should be clear that we must, first of all, proceed to the separation of the heavy and the light degrees of freedom. The subsequent step will be to determine the actual numerical value of the relevant $a_{i}$ coefficients, as a function of the parameters of our underlying theory. Unfortunately, this last step we can do only within the framework of perturbation theory which is suspect in a strongly interacting scalar theory. However, renormalized perturbation theory turns out to be reliable in a trivial theory, as the scalar sector of the MSM appears to be, because the theory is never really strongly interacting [18]. Yet, this is not necessarily so in the presence of additional interactions and fields, particularly supersymmetry, which may alter the ultraviolet properties of the theory. We shall thus rely as much as possible on dimensional and power counting arguments and discuss to what extent these agree with perturbation theory.

Let us begin by reviewing the model in the usual (weakly coupled) linear realization. We have two Higgs doublets $\phi_{1}, \phi_{2}$. For the potential we choose the most general one respecting $C P$ and the discrete symmetry $\phi_{1} \rightarrow-\phi_{1}$, $\phi_{2} \rightarrow \phi_{2}$. Imposing this symmetry automatically avoids an excessive amount of flavor violation [16]. It also suppresses spontaneous $C P$ violation:

$$
\begin{aligned}
V\left(\phi_{1}, \phi_{2}\right)= & \lambda_{1}\left(\phi_{1}^{\dagger} \phi_{1}-v_{1}^{2}\right)^{2}+\lambda_{2}\left(\phi_{2}^{\dagger} \phi_{2}-v_{2}^{2}\right)^{2} \\
& +\lambda_{3}\left[\left(\phi_{2}^{\dagger} \phi_{2}-v_{2}^{2}\right)+\left(\phi_{1}^{\dagger} \phi_{1}-v_{1}^{2}\right)\right]^{2} \\
& +\lambda_{4}\left[\left(\phi_{1}^{\dagger} \phi_{1}\right)\left(\phi_{2}^{\dagger} \phi_{2}\right)-\left(\phi_{1}^{\dagger} \phi_{2}\right)\left(\phi_{2}^{\dagger} \phi_{1}\right)\right] \\
& +\lambda_{6}\left[\operatorname{Im}\left(\phi_{1}^{\dagger} \phi_{2}\right)\right]^{2},
\end{aligned}
$$

where

$$
\phi_{1}=\left(\begin{array}{c}
\alpha_{+} \\
\alpha_{0}
\end{array}\right), \quad \phi_{2}=\left(\begin{array}{c}
\beta_{+} \\
\beta_{0}
\end{array}\right), \quad\left\langle\phi_{1}\right\rangle=\left(\begin{array}{c}
0 \\
v_{1}
\end{array}\right), \quad\left\langle\phi_{2}\right\rangle=\left(\begin{array}{c}
0 \\
v_{2}
\end{array}\right),
$$

$v_{1}, v_{2}$ are real, and $\lambda_{i} \geqslant 0$. We consider the following $2 \times 2$ matrices (as usual $\bar{\phi}=i \tau_{2} \phi^{*}$ )

$$
\Phi_{12}=\left(\bar{\phi}_{1} \phi_{2}\right), \quad \Phi_{21}=\left(\bar{\phi}_{2} \phi_{1}\right), \quad \Phi_{21}=\tau_{2} \Phi_{12}^{*} \tau_{2} .
$$

Under a $\quad \mathrm{SU}_{L}(2) \times \mathrm{U}(1) \quad$ transformation, $\quad \Phi_{i j} \rightarrow$ $\exp [i \bar{\tau} \cdot \bar{\alpha}] \Phi_{i j} \exp \left[-i \tau_{3} \cdot \beta_{3}\right] . \Phi_{12}$ and $\Phi_{21}$ transform in fact in the same way under the larger group $\mathrm{SU}_{L}(2) \times \mathrm{SU}_{R}(2)$, namely, $\Phi_{i j} \rightarrow \exp [i \bar{\tau} \cdot \bar{\alpha}] \Phi_{i j} \exp [-i \bar{\tau} \cdot \bar{\beta}]$. In terms of $\Phi_{i j}$ we define the auxiliary matrices 


$$
I=\Phi_{12}^{\dagger} \Phi_{12}, \quad J=\Phi_{12}^{\dagger} \Phi_{21} .
$$

$I$ and $J$ both transform as $I \rightarrow \exp [i \bar{\tau} \cdot \bar{\beta}] I \exp [-i \bar{\tau} \cdot \bar{\beta}]$. Furthermore,

$$
\langle I\rangle=\frac{v_{1}^{2}+v_{2}^{2}}{2}+\frac{v_{1}^{2}-v_{2}^{2}}{2} \tau_{3}, \quad\langle J\rangle=v_{1} v_{2} .
$$

We would like to point out that other parametrizations are possible. For instance, we could have embedded the two scalar fields in two $2 \times 2$ matrices in this way:

$$
\Phi_{1}=\left(\bar{\phi}_{1} \phi_{1}\right), \quad \Phi_{2}=\left(\bar{\phi}_{2} \phi_{2}\right) .
$$

In this case the $\mathrm{SU}(2) \times \mathrm{SU}(2)$ symmetry is implemented in a slightly different way [19]. However, we choose Eq. (2.3) because Goldstone bosonfields appear quite naturally in the nonlinear parametrization, as we shall see.

In terms of the above matrices the kinetic term reads

$$
T\left(\Phi_{12}\right)=\frac{1}{4} \operatorname{Tr}\left(D_{\mu} \Phi_{12} D^{\mu} \Phi_{12}^{\dagger}\right)
$$

and the potential can be expressed as

$$
\begin{aligned}
V\left(\Phi_{12}, \Phi_{21}\right)= & \frac{\lambda_{1}}{4}\left\{\operatorname{Tr}\left[(I-\langle I\rangle)\left(1+\tau_{3}\right)\right]\right\}^{2} \\
& +\frac{\lambda_{2}}{4}\left\{\operatorname{Tr}\left[(I-\langle I\rangle)\left(1-\tau_{3}\right)\right]\right\}^{2} \\
& +\lambda_{3}\{\operatorname{Tr}[I-\langle I\rangle]\}^{2}+\frac{\lambda_{4}}{4} \operatorname{Tr}\left[I^{2}-\left(I \tau_{3}\right)^{2}\right] \\
& +\frac{\lambda_{6}}{4}\left\{\frac{1}{2 i} \operatorname{Tr}\left[J-J^{\dagger}\right]\right\}^{2} .
\end{aligned}
$$

This potential is invariant under $\mathrm{SU}_{L}(2) \times \mathrm{U}(1)$, but some terms are not invariant under $\mathrm{SU}_{L}(2) \times \mathrm{SU}_{R}(2)$. They break custodial symmetry and may lead, at least potentially, to sizable contributions to the $\rho$ parameter.

After symmetry breaking to $\mathrm{U}(1)_{\mathrm{em}}$, the matrices $I$ and $J$ get a VEV and new fluctuations around the vacuum state appear. Some are massless (the three Goldstone bosons) and other massive (by hypothesis, very massive in our case). We want to separate these very different degrees of freedom to all orders in perturbation theory. The massless degrees of freedom will enter the unitary matrix $U$ and the rest will eventually be integrated out in the coefficients $a_{i}$. The problem is somewhat nontrivial. Suppose, for instance, that, in analogy to the one doublet case, we write the $2 \times 2$ matrices $\Phi_{12}$ and $\Phi_{21}$ as the product of a unitary matrix and an Hermitian one; e.g.,

$$
\Phi_{12}=\mathcal{U} H_{12} .
$$

Then the unitary matrix $\mathcal{U}=\exp (i \theta / v) \exp (i \bar{\tau} \bar{G} / v)$, where $v^{2}$ $=\left(v_{1}^{2}+v_{2}^{2}\right) / 2$ is the combination of vacuum expectation values (VEV's) relevant for the $W$ mass, would hopefully collect the Goldstone bosons and $H_{12}=(\sigma 1+\bar{\tau} \cdot \bar{\gamma})$ would be the extension to the two doublet case of $\sigma 1$ (notice the appearance of the additional phase $\theta$ in the 2HDM). Unfortunately, this separation is far too naive. In fact, although the $\bar{G}$ fields do not appear in the scalar potential, they mix with the fields in $H$ due to the kinetic term and, therefore, cannot be identified as Goldstone bosons.

Yet the above decomposition is quite suggestive because when one substitutes back in the scalar potential any decomposition of the form $\Phi_{12}=U M_{12}$, where $M_{12}$ is not necessarily Hermitian, $U$ drops from the potential exactly. Thus instead of assuming that $M$ is Hermitian, we shall allow for a more general matrix (this is just fine, as long as the decomposition is still unique). We single out the Goldstone bosons by making an infinitesimal gauge transformation specialized to the broken generators:

$$
\begin{aligned}
\delta_{\varepsilon}= & +i \bar{T}^{L} \cdot \bar{\epsilon}^{L}+i T^{R} \epsilon^{R}=1+i T_{+}^{L} G_{+}+i T_{-}^{L} G_{-} \\
& +i \frac{T_{3}^{L}-T_{3}^{R}}{2} G_{0} .
\end{aligned}
$$

Acting with such a transformation on the vacuum configuration for, e.g., $\Phi_{12}$, we obtain

$$
\delta_{\epsilon} \Phi_{12}=\left(\begin{array}{cc}
v_{1}+i G_{0} \frac{v_{1}}{v} & i \sqrt{2} G_{+} \frac{v_{2}}{v} \\
i \sqrt{2} G_{-} \frac{v_{1}}{v} & v_{2}-i G_{0} \frac{v_{2}}{v}
\end{array}\right) .
$$

And an analogous expression for $\Phi_{21}$. Goldstone bosons and massive excitations must be orthonormal for the kinetic terms to be diagonal. Once the former have been identified, the latter are uniquely determined. We obtain

$$
\Phi_{12}=\left(\begin{array}{cc}
\operatorname{Re}\left[\alpha_{0}\right]+i\left(G_{0} \frac{v_{1}}{v}+A_{0} \frac{v_{2}}{2}\right) & \sqrt{2}\left(H_{+} \frac{v_{1}}{v}+i G_{+} \frac{v_{2}}{v}\right) \\
\sqrt{2}\left(H_{-} \frac{v_{2}}{2}+i G_{-} \frac{v_{1}}{v}\right) & \operatorname{Re}\left[\beta_{0}\right]+i\left(-G_{0} \frac{v_{2}}{v}+A_{0} \frac{v_{1}}{v}\right)
\end{array}\right) .
$$


Keeping terms at most linear in the fields, $\Phi_{12}$ can also be written as

$$
\Phi_{12}=\exp \left[i \frac{\bar{G} \cdot \bar{\tau}}{v}\right]\left(\begin{array}{cc}
\operatorname{Re}\left[\alpha_{0}\right]+i A_{0} \frac{v_{2}}{v} & \sqrt{2} H_{+} \frac{v_{1}}{v} \\
\sqrt{2} H_{-} \frac{v_{2}}{v} & \operatorname{Re}\left[\beta_{0}\right]+i A_{0} \frac{v_{1}}{v}
\end{array}\right) .
$$

Notice that an alternative form for $\Phi_{12}$, useful for calculations, is

$$
\Phi_{12}=\exp \left[i \frac{\bar{G} \cdot \bar{\tau}}{v}\right]\left(\sigma+i A_{0}+\bar{\tau} \bar{H}\right)\left(\begin{array}{cc}
\frac{v_{2}}{v} & 0 \\
0 & \frac{v_{1}}{v}
\end{array}\right),
$$

with

$$
\begin{gathered}
\sigma=\frac{1}{2 \sqrt{2}}\left(\frac{\operatorname{Re}\left[\alpha_{0}\right]}{\sin \beta}+\frac{\operatorname{Re}\left[\beta_{0}\right]}{\cos \beta}\right), \\
H_{3}=\frac{1}{2 \sqrt{2}}\left(\frac{\operatorname{Re}\left[\alpha_{0}\right]}{\sin \beta}-\frac{\operatorname{Re}\left[\beta_{0}\right]}{\cos \beta}\right) .
\end{gathered}
$$

This is the expression we were after. It satisfies the following properties: (1) It is a parametrization of $\Phi_{12} ;(2)$ it is of the form $\Phi_{12}=U M_{12}$, where $U \in \mathrm{SU}(2)$; (3) it diagonalizes the kinetic terms by construction; (4) it can be proven to be unique.

With this parametrization we also have

$$
\begin{aligned}
\Phi_{21}= & \tau_{2} \Phi_{12}^{*} \tau_{2}=U M_{21}=\exp \left[i \frac{\bar{G} \cdot \bar{\tau}}{v}\right]\left(\sigma-i A_{0}-\bar{\tau} \bar{H}\right) \\
& \times\left(\begin{array}{cc}
\frac{v_{1}}{v} & 0 \\
0 & \frac{v_{2}}{v}
\end{array}\right)
\end{aligned}
$$

We now plug the above decomposition $\Phi_{12}=U M_{12}$ into the kinetic term (2.7)

$$
T=\frac{1}{4} \operatorname{Tr}\left[D_{\mu}\left(U M_{12}\right)\left(D_{\mu}\left(U M_{12}\right)\right)^{\dagger}\right]
$$

$M_{21}$ does not appear here, but it does in the potential terms.

Naively, setting the masses of all heavy particles to infinity would take us to the minimum of the potential, $M_{12}$ $=\left\langle M_{12}\right\rangle$. Plugging this back in Eq. (2.7), and using $\operatorname{Tr}\left(\tau_{3} D_{\mu} U D^{\mu} U^{\dagger}\right)=0$ we recover at the classical level the lowest-dimensional term $v^{2} \operatorname{Tr}\left(D_{\mu} U D^{\mu} U^{\dagger}\right)$ in the ECL. Letting $M_{12} \rightarrow\left\langle M_{12}\right\rangle$ and $D_{\mu} \rightarrow \partial_{\mu}$ we obtain the kinetic terms

$$
\frac{1}{v^{2}} \partial_{\mu} \bar{G} \partial^{\mu} \bar{G} \operatorname{Tr}\left(\left\langle M_{12}\right\rangle\left\langle M_{12}\right\rangle^{\dagger}\right)+\operatorname{Tr}\left(\partial_{\mu} M_{12} \partial^{\mu} M_{12}^{\dagger}\right)
$$

The crossed term vanishes:

$$
\begin{aligned}
& \frac{i}{v} \operatorname{Tr}\left[\bar{\tau} \partial_{\mu} \bar{G}\left(\left\langle M_{12}\right\rangle \partial^{\mu} M_{12}^{\dagger}-\partial^{\mu} M_{12}\left\langle M_{12}\right\rangle^{\dagger}\right)\right] \\
& \quad=2 \frac{v_{1} v_{2}}{v^{2}} \operatorname{Tr}\left(\partial_{\mu} \bar{G} \bar{\tau} \partial^{\mu} A_{0}\right)=0 .
\end{aligned}
$$

We are then left with the canonical diagonal kinetic terms for the fields $\bar{G}, A_{0}, H_{1}^{0}, H_{2}^{0}, H_{+}, H_{-}$. The fields $H_{1}^{0}$ and $H_{2}^{0}$ are the mass eigenvalues of the mass matrix in the $C P$-even neutral sector. This leads to the appearance of the mixing angle $\alpha$ which depends on the parameters of the potential

$$
\begin{gathered}
H_{1}^{0}=\frac{\operatorname{Re}\left[\alpha_{0}\right]}{\sqrt{2}} \cos \alpha+\frac{\operatorname{Re}\left[\beta_{0}\right]}{\sqrt{2}} \sin \alpha, \\
H_{2}^{0}=-\frac{\operatorname{Re}\left[\alpha_{0}\right]}{\sqrt{2}} \sin \alpha+\frac{\operatorname{Re}\left[\beta_{0}\right]}{\sqrt{2}} \cos \alpha .
\end{gathered}
$$

Neither $H_{1}^{0}$ nor $H_{2}^{0}$ have simple interaction terms. In particular, both have nonvanishing VEV's. The combinations

$$
\begin{aligned}
& S=\sin (\alpha-\beta) H_{1}^{0}+\cos (\alpha-\beta) H_{2}^{0}, \\
& H=\cos (\alpha-\beta) H_{1}^{0}-\sin (\alpha-\beta) H_{2}^{0},
\end{aligned}
$$

on the other hand, "diagonalize" the interaction pieces in the Lagrangian. By this we mean that the field $H$ (which has $\langle H\rangle=v$ ) has exactly the same interaction terms as the standard Higgs boson would have, in particular the coupling $g M_{W} H W^{+} W^{-}$, characteristic of a spontaneously broken theory (yet $H$ is not an eigenstate of the mass matrix, as mentioned). On the other hand, the field $S$ (with $\langle S\rangle=0$ ) does not have any couplings of the above form. This observation turns out to be important to understand our results.

Although classically $a_{0}, a_{1}$, and $a_{8}$ vanish in the limit where all scalar particles are very massive, at the quantum level these coefficients will be generically nonzero. To obtain their true value we must integrate out the fields contained in the matrices $M_{12}$ and $M_{21}$

Since it accompanies a custodially breaking operator, $a_{0}$ must on symmetry grounds be proportional to a typical mass splitting or a custodial-breaking parameter, such as $g^{\prime 2}$. Naively,

$$
a_{0} \sim\left\{\frac{g^{2}}{16 \pi^{2}} \frac{\Delta M^{2}}{M_{W}^{2}}, \frac{g^{\prime 2}}{16 \pi^{2}} \ln \frac{M_{s}^{2}}{M_{W}^{2}}\right\},
$$

where $M_{s}$ is a typical heavy-scalar mass. In fact, we will see that the dependence on the quadratic mass splittings, $\Delta M^{2}$ $=M^{2}-M_{s}^{2}$, is quadratic and not linear. At any rate nondecoupling effects may be important if large mass splittings are present. (As is well known, the Appelquist-Carazzone decoupling theorem does not go through for spontaneously broken theories [20].) Other potential nondecoupling effects are contained in the coefficients of the $O\left(p^{4}\right)$ operators. On dimensional grounds these coefficients will be of order

$$
a_{i} \sim \frac{1}{16 \pi^{2}}\left[\ln \frac{M_{s}^{2}}{M_{W}^{2}}+c+O\left(\frac{\Delta M^{2}}{M_{s}^{2}}\right)\right] \quad(i \neq 0) .
$$


$c$ is a finite constant and $M_{s}$ is the mass of the $C P$-odd scalar, taken as reference scale. These terms will be less important at low energies since it is clear that the leading pieces in the momentum expansion are contained in the $d$ $=2$ terms. At energies $q^{2} \ll 16 \pi^{2} v^{2}$ the $O\left(p^{4}\right)$ operators will be suppressed with respect to the $O\left(p^{2}\right)$ ones, although they rapidly become important as the energy increases.

At this point we should reexamine the field contents of our theory in the nonlinear realization. We have three Goldstone bosons collected in the unitary matrix $U$, two charged Higgs bosons $H_{+}$and $H_{-}$, two $C P$-even neutral fields $\sigma$ and $H_{3}$ or, equivalently, $H_{1}^{0}, H_{2}^{0}$, and the $C P$-odd neutral $A_{0}$. All of them are supposed to be heavy.

\section{THE EFFECTIVE LAGRANGIAN COEFFICIENTS}

The part of the $O\left(p^{4}\right)$ effective Lagrangian relevant for the determination of the oblique corrections is the one given in Eq. (1.3). The corresponding coefficients contain the traces of the underlying theory accessible at experiments much below the energy scales $M_{s}$ or $4 \pi v$, whichever is smaller.

It is customary to parametrize possible departures from the MSM with the parameters $\varepsilon_{1}, \varepsilon_{2}$, and $\varepsilon_{3}$ [9]. These parameters, in the limit of vacuum polarization dominance, coincide with the $e_{i}$ parameters defined by

$$
\begin{gathered}
\frac{1}{M_{W}^{2}}\left[A_{33}(0)-A_{W W}(0)\right] \equiv e_{1}, \\
F_{W W}\left(M_{W}^{2}\right)-F_{33}\left(M_{W}^{2}\right) \equiv e_{2}, \\
\frac{c}{S} F_{30}\left(M_{Z}^{2}\right) \equiv e_{3}
\end{gathered}
$$

in terms of the vacuum polarization amplitudes

$$
\Pi_{\mu \nu}^{i j}(q)=-i g_{\mu \nu}\left[A^{i j}(0)+q^{2} F^{i j}\left(q^{2}\right)\right]+q_{\mu} q_{\nu} \text { terms, }
$$

where $i, j=W, 0,3$, stand for the $W^{ \pm}, B$, and $W^{3}$ gauge boson fields, respectively. The $S, T$, and $U$ parameters [8] are trivially related to the above.

In an effective theory, such as the one described by the Lagrangians (1.2) and (1.3), $\varepsilon_{1}, \varepsilon_{2}$, and $\varepsilon_{3}$ receive one-loop contributions from the leading $O\left(p^{2}\right)$ term $v^{2} \operatorname{Tr}\left(D_{\mu} U D^{\mu} U^{\dagger}\right)$ and the tree level contributions from the $a_{i}$. Thus

$$
\begin{gathered}
\varepsilon_{1}=2 a_{0}+\cdots, \quad \varepsilon_{2}=-g^{2} a_{8}+\cdots, \\
\varepsilon_{3}=-g^{2} a_{1}+\cdots,
\end{gathered}
$$

where the ellipses symbolize the one-loop $O\left(p^{2}\right)$ contributions. The latter are totally independent of the specific symmetry-breaking sector. The nonlinear nature of $\mathcal{L}^{2}$ induces new divergences which are absorbed by a proper redefinition of the $a_{i}$. These divergences are by construction independent of the underlying theory, so we automatically know the logarithmic dependence of the coefficients for any two Higgs doublet model $[13,14]$ :

$$
\begin{gathered}
a_{0} \sim \frac{g^{\prime 2}}{16 \pi^{2}} \frac{3}{8} \ln \frac{M_{W}^{2}}{M_{s}^{2}}, \\
a_{1} \sim \frac{1}{16 \pi^{2}} \frac{1}{12} \ln \frac{M_{W}^{2}}{M_{s}^{2}}, \\
a_{8} \sim 0 .
\end{gathered}
$$

By construction this logarithmic dependence is exact, even in the nonperturbative large $M_{s}$ limit. The above are renormalized coefficients in the modified minimal subtraction scheme $(\overline{\mathrm{MS}})$ scheme.

The coefficients $a_{i}$ contain, in addition, constant and $O\left(\Delta M^{2} / M_{s}^{2}\right)$ corrections. These subleading contributions are nonuniversal and have to be determined by matching, e.g., the renormalized self-energies, or an appropriate combination thereof, between fundamental and effective theories. For instance, we can match the combinations of self-energies appearing in Eq. (3.1). When we compute the values of the $a_{i}$ coefficients via the matching conditions, most of the diagrams cancel between both sides of the matching equation. Only those containing at least one heavy particle contribute, properly expanded in $p^{2}$, to the coefficients of the ECL.

The values of the $\varepsilon_{i}$ in a $2 \mathrm{HDM}$ have already been calculated in the past [12] in the linear perturbative regime. In the nonlinear realization there are differences already at the level of Feynman rules, and some simplifications worth pointing out. For instance, the vertex with one scalar neutral Higgs boson $H_{3}$, one charged Goldstone boson $G_{+}$, and one gauge boson $W_{\mu}^{-}$, is given by $g H_{3} \partial_{\mu} G_{+} W_{\mu}^{-}$in the nonlinear case, and by $(g / 2) H_{3} \overleftrightarrow{\partial}_{\mu} G_{+} W_{\mu}^{-}$in the linear parametrization.

We are interested only in the leading corrections in the limit $q^{2} \approx M_{W}^{2} \ll M_{s}$, where $M_{s}$ is a typical heavy-scalar mass. Then we can set $M_{W}=M_{G}=0 \quad\left(M_{G}\right.$ is the gaugedependent Goldstone bosons mass) in the internal lines. Moreover, when calculating the diagrams contributing to $e_{2}$ and $e_{3}$, those with gauge bosons in the internal lines do not contribute. These simplifications follow from simpledimensional considerations. For instance, the diagrams containing one internal vector-boson line are proportional to $g^{2} M_{W}^{2}$ and their overall contribution to $e_{3}$ is ultraviolet finite. Since $e_{3}$ is proportional to the dimensionless derivatives of the vacuum polarization, there must be a $M_{s}^{2}$ in the denominator, and so the contribution is proportional to $M_{W}^{2} / M_{s}^{2}$ and, therefore, subleading. In the same way it is possible to see by inspection that some diagrams (such as tadpoles) do not contribute in the matching relations for $e_{1}$. We are left with the diagrams in Fig. 1 for the matching relations for $e_{1}, e_{2}$, and $e_{3}$. The only nonzero masses in these diagrams are the heavy-scalar masses.

If we neglect terms of $O\left(M_{W}^{2} / M_{s}^{2}\right)$ the values one obtains from Eq. (A3) for the $a_{i}$ coefficients are

$$
\begin{aligned}
a_{0}= & \frac{g^{2}}{16 \pi^{2}} \frac{1}{24} \frac{\Delta_{+}^{2}\left(\Delta_{+}^{2}-s^{2} \Delta_{1}^{2}-c^{2} \Delta_{2}^{2}\right)}{M_{s}^{2} M_{W}^{2}}+\frac{g^{\prime 2}}{16 \pi^{2}} \frac{3}{8} \\
& \times\left[\ln \frac{M_{W}^{2}}{M_{s}^{2}}+\frac{5}{6}+O\left(\frac{\Delta_{i}^{2}}{M_{s}^{2}}\right)\right],
\end{aligned}
$$



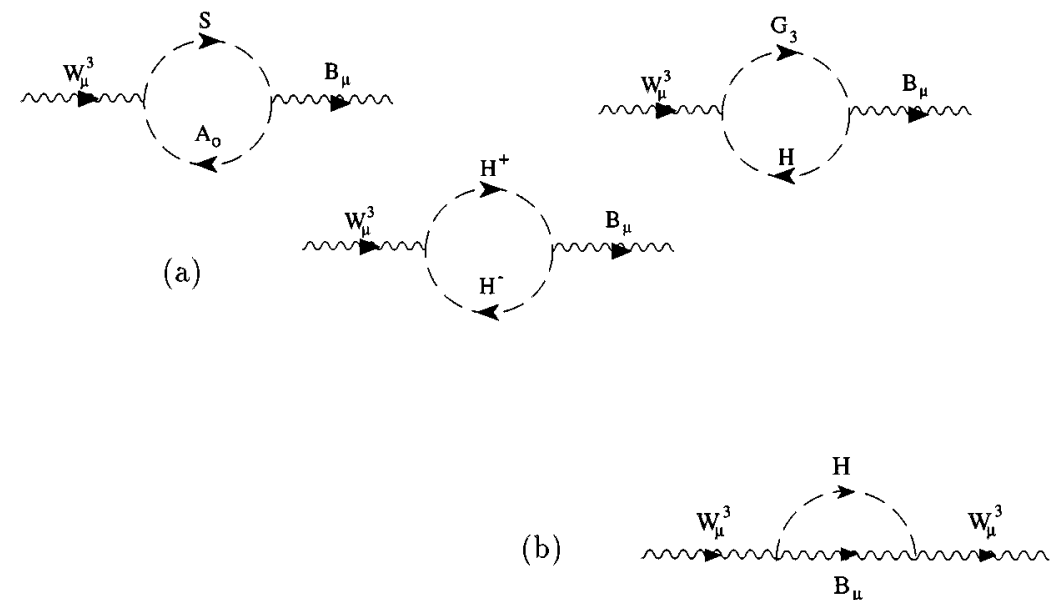

(c)

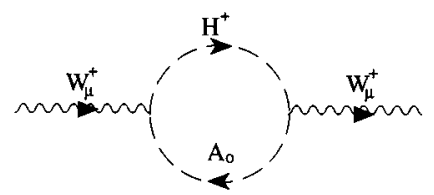

$$
\begin{gathered}
a_{1}=\frac{1}{16 \pi^{2}} \frac{1}{12}\left[\ln \frac{M_{W}^{2}}{M_{s}^{2}}+\frac{5}{6}+O\left(\frac{\Delta_{i}^{2}}{M_{s}^{2}}\right)\right], \\
a_{8}=O\left(\frac{1}{16 \pi^{2}} \frac{\Delta_{i}^{2}}{M_{s}^{2}}\right) .
\end{gathered}
$$

These expressions are valid in the limit $M_{W}^{2} \lesssim \Delta_{i}^{2} \ll M_{s}^{2}$. Here $s^{2}$ and $c^{2}$ are the sinus and cosinus of $\alpha-\beta$, where $\alpha$ is the angle that mixes the two neutral scalars and $\tan \beta=v_{2} / v_{1}$. The quantities $\Delta_{i}^{2}$ denote the quadratic mass splittings of the scalar labeled $i(i=+,-$ correspond to the charged Higgs bosons, $i=1,2$ to the two $C P$-even neutrals) with respect to the $C P$-odd neutral scalar, whose mass is taken as reference scale; that is $\Delta_{i}^{2} \equiv M_{i}^{2}-M_{s}^{2}$. In the above expressions we have kept the dominant terms only.

The first observation is that the constant pieces in Eqs. (3.7)-(3.9) are the same ones as those obtained in $[15,21,22]$ in a similar calculation in the MSM, with the obvious replacement $M_{s} \rightarrow M_{H}$. Some nondecoupling effects do remain in the 2HDM, but they are identical to the MSM ones. Thus in the limit where the mass splittings are negligible compared to the typical scale in the symmetry-breaking sector the two Higgs doublet model cannot be distinguished by any low-energy experiment from the minimal standard model. For instance, the combination of coefficients
FIG. 1. Feynman diagrams required to evaluate the coefficients $a_{i}$ via matching conditions for $\varepsilon_{i}$. Diagrams (a) are for $\varepsilon_{3}$, diagrams (c) for $\varepsilon_{2}$, and [diagrams (c) +diagram (b)] for $\varepsilon_{1}$. which is zero in the MSM in the limit of a heavy Higgs boson mass, is still zero in the $2 \mathrm{HDM}$ provided that $\Delta_{i}^{2}$ $\ll M_{s}^{2}$. (The above combination corresponds to straight lines in the $\Gamma_{l}, \sin _{\mathrm{eff}}^{2} \theta_{W}$ plane. See [15].)

Can we understand this? As discussed in [20,22], the source of the nondecoupling effects can be traced back to the appearance of mixed heavy or light vertices without derivative couplings. These are characteristic of spontaneously broken theories (in which one shifts some field). An archetypical example has already been mentioned: the vertex $g M_{W} H W^{+} W^{-}$, but also vertices such as $g H G^{+} W^{-}$. The point is that, once we write the neutral scalar sector in terms of the $H$ and $S$ fields, these couplings are identical to the ones in the MSM. $H$ is the only culprit of nondecoupling effects, while fields such as $S, H_{+}, H_{-}$, and $A_{0}$ leave, after integrating them out, contributions suppressed by powers of heavy masses, i.e., they decouple.

A second remark is that the dependence of $a_{0}$ on the quadratic mass splittings is of order $g^{2}\left(\Delta_{i}^{2}\right)^{2} / M_{W}^{2} M_{s}^{2}$ or $g^{\prime 2}\left(\Delta_{i}^{2}\right) / M_{s}^{2}$. Once we understand that nondecoupling effects must be the same in the limit $\Delta_{i}^{2} / M_{s}^{2} \rightarrow 0$ as in the MSM, it is clear that the naive counting in Eq. (2.22) cannot 
hold. We need an additional power of $M_{s}^{2}$ in the denominator, and that forces $\Delta_{i}^{2}$ to appear quadratically.

What happens beyond perturbation theory? This we cannot answer precisely, of course. But we can stand by the order of magnitude estimates derived from the general arguments given above. We cannot prove that the constant pieces in $a_{1}$, for instance, will be the same after a nonperturbative calculation. However, we can certainly conjecture that the nondecoupling pieces (those not suppressed by inverse powers of $M_{s}^{2}$ ) will be the same as those in the MSM. And for the latter, perturbation theory turns out to be eventually reliable as previously discussed.

Some nonstandard nondecoupling effects remain, however, in the case where the mass splittings are sizable, $\Delta_{i}^{2}$ $\sim M_{s}^{2}$. To discuss to what extent these effects are visible it is best to return to the $\varepsilon_{i}$ parameters.

\section{COMPARISON WITH EXPERIMENT AND CONCLUSIONS}

We now compare the results on $\varepsilon_{i}$ parameters with the experimental data from LEP and SLAC Large Detector (SLD). Our aim is to restrict the allowed parameter space in the sort of models we are considering. If we set a maximum value $\Delta^{2}$ for all the quadratic mass splittings, $\left|\Delta_{i}^{2}\right|<\Delta^{2}$, we obtain from Eq. (A3) that the $\varepsilon_{i}$ parameters lie between two extreme values given below. To ease the comparison with the MSM we quote below the deviations with respect to the values for $\varepsilon_{i}$ obtained there. This is the reason why the value of $M_{H}$ (the Higgs boson mass in the MSM) appears in the expressions

$$
\begin{aligned}
& \delta \varepsilon_{1}^{\max } \approx-\frac{3}{4} \frac{g^{\prime 2}}{16 \pi^{2}} \ln \frac{M_{s}^{2}}{M_{H}^{2}}+\frac{1}{16 \pi^{2}} \frac{\Delta^{2}}{M_{s}^{2}}\left(\frac{g^{2}}{6} \frac{\Delta^{2}}{M_{W}^{2}}+\frac{3}{4} g^{\prime 2}\right. \\
&\left.+\frac{3}{8} g^{\prime 2} \frac{\Delta^{2}}{M_{s}^{2}}\right), \\
& \delta \varepsilon_{1}^{\min } \approx-\frac{3}{4} \frac{g^{\prime 2}}{16 \pi^{2}} \ln \frac{M_{s}^{2}}{M_{H}^{2}}-\frac{1}{16 \pi^{2}} \frac{\Delta^{2}}{M_{s}^{2}}\left(\frac{g^{2}}{48} \frac{\Delta^{2}}{M_{W}^{2}}+\frac{3}{4} g^{\prime 2}\right. \\
&\left.-\frac{3}{8} g^{\prime 2} \frac{\Delta^{2}}{M_{s}^{2}}\right), \\
& \delta \varepsilon_{2}^{\max } \approx \frac{g^{2}}{16 \pi^{2}} \frac{1}{240}\left(\frac{\Delta^{2}}{M_{s}^{2}}\right)^{2},(4.1 \mathrm{a}) \\
& \delta \varepsilon_{2}^{\min } \approx-\frac{g^{2}}{16 \pi^{2}} \frac{1}{30}\left(\frac{\Delta^{2}}{M_{s}^{2}}\right)^{2}, \\
& \frac{g^{2}}{16 \pi^{2}} \frac{1}{12} \ln \frac{M_{s}^{2}}{M_{H}^{2}}+\frac{g^{2}}{16 \pi^{2}} \frac{5}{24} \frac{\Delta^{2}}{M_{s}^{2}}-\frac{g^{2}}{16 \pi^{2}} \frac{1}{80}\left(\frac{\Delta^{2}}{M_{s}^{2}}\right)^{2}, \\
& \delta \varepsilon_{3}^{\min } \approx-\frac{g^{2}}{16 \pi^{2}} \frac{1}{12} \frac{\Delta^{2}}{M_{s}^{2}}+\frac{g^{2}}{16 \pi^{2}} \frac{1}{24}\left(\frac{\Delta^{2}}{M_{s}^{2}}\right)^{2} . \quad(4.1 \mathrm{~d})
\end{aligned}
$$

Expressions (4.1a)-(4.1d) are the exact maxima and minima of the corresponding expressions given in Eq. (A3). The ex-

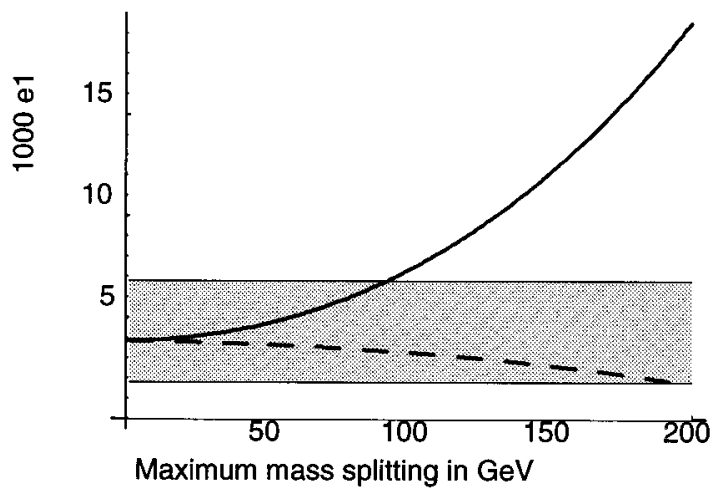

FIG. 2. Maximum (continuous line) and minimum (dashed line) possible values for $10^{3} \times \varepsilon_{1}$ in the $2 \mathrm{HDM}$ as a function of the largest scalar linear mass splitting $\Delta_{\max }$. The grey zone corresponds to the allowed experimental values.

pressions for $\delta \varepsilon_{3}^{\max , \min }$ are, however, simplified approximations to the corresponding maximum and minimum of Eq. (A3). Terms of order $\left(\Delta^{2} / M_{s}^{2}\right)^{3}$ and higher have been neglected in all cases. Note that these expressions do not depend at all on $\sin (\alpha-\beta)$. Moreover, we checked that they differ from the actual minimum obtained from Eqs. (A1) by less than $5 \%$ if the mass splittings are less than $150 \mathrm{GeV}$.

The extraction of the $\varepsilon_{i}$ parameters from experiment can be done, e.g., along the lines of [23]. Using the latest experimental data from LEP [5], we obtain [24]

$$
\begin{gathered}
\varepsilon_{1}=(4.7 \pm 1.3) \times 10^{-3}, \\
\varepsilon_{2}=(-7.8 \pm 3.3) \times 10^{-3}, \\
\varepsilon_{3}=(4.8 \pm 1.4) \times 10^{-3} .
\end{gathered}
$$

$\varepsilon_{1}$ is the most restrictive parameter due to its strong dependence on the splittings and we focus our attention on it. In Fig. 2 we plot maximum and minimum values for $\varepsilon_{1}$ in function of the maximally allowed linear mass splitting $\Delta_{\max } \approx \Delta^{2} / 2 M_{s}$ (that is, $\left|M_{i}-M_{s}\right|<\Delta_{\max }$ for all $i$ ). These values for $\varepsilon_{1}$ are obtained by adding the MSM contribution as taken from [23] and our expressions (4.1a), (4.1b). A value for $M_{H}$ in the MSM of $300 \mathrm{GeV}$ was chosen, but the graph itself is, of course, independent of the particular value of $M_{H}$ one chooses. Also, the experimentally allowed values for $\varepsilon_{1}$ are shown; it is easy to see that splittings of the order of $100 \mathrm{GeV}$ or more around a reference mass of approximately $600 \mathrm{GeV}$ are perfectly allowed.

In conclusion, we have analyzed the situation in which the symmetry-breaking sector of the SM consists in two scalar doublets with masses in the $\mathrm{TeV}$ region. We have separated the light and heavy degrees of freedom and constructed an effective chiral Lagrangian for the former. The information about the latter is contained in a few low-energy coefficients. We have shown that these coefficients can be calculated in terms of a few Feynman diagrams (see Fig. 1). We have found that the models exhibits nondecoupling effects; that is, nonzero values for the coefficients of operators with dimensionality $d \leqslant 4$ even in the $M_{s} \rightarrow \infty$ limit. These nondecoupling effects in the limit of exact custodial symmetry are exactly the same ones as those in the MSM. We have analyzed which restrictions current data set on two Higgs doublet models; due to the equivalence between the two Higgs 
doublet model and the MSM in the limit of exact custodial symmetry in the scalar potential the current bounds are very weak.

\section{ACKNOWLEDGMENTS}

We would like to thank M. J. Herrero for discussions concerning possible nondecoupling effects in supersymmetric theories, A. Andrienov for discussions about the two Higgs doublets model, and V. Koulovassilopoulos for several comments regarding the manuscript. P.C. acknowledges financial support from INFN, Frascati, Italy. This research was partially supported by Grant Nos. ERB-CHRXCT930343 (E.U.), AEN95-0590 (CICYT), and GRQ93-1047 (CIRIT).

\section{APPENDIX: FORMULAS FOR $\varepsilon_{i}$ PARAMETERS}

We present here the exact result in the 2HDM to the $\delta \varepsilon_{i}$ parameters at one loop, defined as $\delta \varepsilon_{i}=\varepsilon_{i}^{2 \mathrm{HDM}}$ $-\varepsilon_{i}^{\mathrm{MSM}}$ :

$$
\begin{aligned}
\delta \varepsilon_{1}= & \frac{g^{2}}{16 \pi^{2}} \frac{1}{4 M_{w}^{2}}\left[s^{2} f\left(M_{H^{+}}^{2}, M_{H_{2}^{0}}^{2}\right)+c^{2} f\left(M_{H^{+}}^{2}, M_{H_{1}^{0}}^{2}\right)\right. \\
& \left.+f\left(M_{H^{+}}^{2}, M_{A^{0}}^{2}\right)-s^{2} f\left(M_{A^{0}}^{2}, M_{H_{2}^{0}}^{2}\right)-c^{2} f\left(M_{A^{0}}^{2}, M_{H_{1}^{0}}^{2}\right)\right] \\
& -\frac{g^{\prime 2}}{16 \pi^{2}} \frac{3}{4}\left(s^{2} \ln \left[\frac{M_{H_{2}^{0}}^{2}}{M_{H}^{2}}\right]+c^{2} \ln \left[\frac{M_{H_{1}^{0}}^{2}}{M_{H}^{2}}\right]\right), \\
\delta \varepsilon_{2}= & -\frac{g^{2}}{16 \pi^{2}} \frac{1}{12}\left[s^{2} g\left(M_{H^{+}}, M_{H_{2}^{0}}\right)+c^{2} g\left(M_{H^{+}}^{2}, M_{H_{1}^{0}}^{2}\right)\right. \\
& +g\left(M_{H^{+}}^{2}, M_{A^{0}}^{2}\right)-s^{2} g\left(M_{A^{0}}^{2}, M_{H_{2}^{0}}^{2}\right)-c^{2} g\left(M_{\left.\left.A^{0}, M_{H_{1}^{0}}^{2}\right)\right],}^{2}\right) \\
\delta \varepsilon_{3}= & \frac{g^{2}}{16 \pi^{2}} \frac{1}{12}\left\{s ^ { 2 } g \left(M_{A^{0}, M_{H_{1}^{0}}}^{2}+c^{2} g\left(M_{\left.A^{0}, M_{H_{2}^{0}}\right)}\right.\right.\right. \\
& +\frac{1}{2} \ln \left[\frac{M_{A^{0}}^{2}}{M_{H}^{2}}\right]-\ln \left[\frac{M_{H^{+}}^{2}}{M_{H}^{2}}\right]+\left(\frac{1+c^{2}}{2}\right) \ln \left[\frac{M_{H_{1}^{0}}^{2}}{M_{H}^{2}}\right] \\
& \left.+\left(\frac{1+s^{2}}{2}\right) \ln \left[\frac{M_{H_{2}^{0}}^{2}}{M_{H}^{2}}\right]\right\},
\end{aligned}
$$

where

$$
\begin{gathered}
f(a, b) \equiv \frac{a b}{a-b} \ln \left[\frac{b}{a}\right]+\frac{a+b}{2}, \\
g(a, b) \equiv-\frac{5}{6}+\frac{2 a b}{(a-b)^{2}}+\frac{(a+b)\left(a^{2}-4 a b+b^{2}\right)}{2(a-b)^{3}} \ln \frac{a}{b} .
\end{gathered}
$$

Here $s^{2} \equiv \sin ^{2}(\alpha-\beta)$ where $\alpha$ is the angle that mixes the two neutral scalars, and $\tan \beta=v_{2} / v_{1} . M_{H}$ is the MSM Higgs boson mass. These results coincide with the ones previously published [12]. We obtained them in the Feynman-'t Hooft gauge with linear gauge fixing [15]. Since we are interested in the large scalar masses limit, we set a reference mass $M_{s}=M_{A^{0}}$, assumed large, and expand functions (A2) in the quadratic mass splittings. Defining $\Delta_{i}^{2} \equiv M_{i}^{2}-M_{s}^{2}$, we obtain

$$
\begin{aligned}
& \delta \varepsilon_{1} \approx \frac{g^{2}}{16 \pi^{2}} \frac{1}{12} \frac{\Delta_{+}^{2}\left(\Delta_{+}^{2}-s^{2} \Delta_{1}^{2}-c^{2} \Delta_{2}^{2}\right)}{M_{s}^{2} M_{w}^{2}}-\frac{3}{4} \frac{g^{\prime 2}}{16 \pi^{2}} \ln \frac{M_{s}^{2}}{M_{H}^{2}} \\
&-\frac{3}{4} \frac{g^{\prime 2}}{16 \pi^{2}} c^{2} \ln \left(1+\frac{\Delta_{1}^{2}}{M_{s}^{2}}\right)-\frac{3}{4} \frac{g^{\prime 2}}{16 \pi^{2}} s^{2} \ln \left(1+\frac{\Delta_{2}^{2}}{M_{s}^{2}}\right), \\
& \delta \varepsilon_{2} \approx-\frac{g^{2}}{16 \pi^{2}} \frac{1}{60} \frac{\Delta_{+}^{2}\left(\Delta_{+}^{2}-s^{2} \Delta_{1}^{2}-c^{2} \Delta_{2}^{2}\right)}{M_{s}^{4}}, \\
& \delta \varepsilon_{3} \approx \frac{g^{2}}{16 \pi^{2}} \frac{1}{120} \frac{s^{2}\left(\Delta_{1}^{2}\right)^{2}+c^{2}\left(\Delta_{2}^{2}\right)^{2}}{M_{s}^{4}}+\frac{g^{2}}{16 \pi^{2}} \frac{1}{12} \\
& \times\left[\ln \frac{M_{s}^{2}}{M_{H}^{2}}+\frac{1+c^{2}}{2} \ln \left(1+\frac{\Delta_{1}^{2}}{M_{s}^{2}}\right)+\frac{1+s^{2}}{2} \ln \left(1+\frac{\Delta_{2}^{2}}{M_{s}^{2}}\right)\right. \\
&\left.-\ln \left(1+\frac{\Delta_{+}^{2}}{M_{s}^{2}}\right)\right] .
\end{aligned}
$$

[1] D. Toussaint, Phys. Rev. D 18, 1626 (1978); H. Georgi, Hadr. J. Phys. 1, 155 (1978).

[2] R. Peccei and H. Quinn, Phys. Rev. D 16, 1791 (1977).

[3] S. Weinberg, Phys. Rev. Lett. 63, 2333 (1989); Phys. Rev. D 42, 860 (1990); Y.-L. Wu and L. Wolfenstein, Phys. Rev. Lett. 73, 1762 (1994).

[4] S. L. Glashow and S. Weinberg, Phys. Rev. D 15, 1958 (1977); E. Paschos, ibid. 15, 1966 (1977); J. Liu and L. Wolfenstein, Nucl. Phys. B289, 1 (1987); L. J. Hall and S. Weinberg, Phys. Rev. D 48, R979 (1993).
[5] Internal Note Warsaw 96 from LEP Electroweak Working Group, LEP-EWWG 96-02.

[6] See, e.g., T. Appelquist, N. Evans, and S. Selipsky, Phys. Lett. B 374, 145 (1996).

[7] T. Appelquist and J. Carazzone, Phys. Rev. D 11, 2856 (1975).

[8] M. Peskin and T. Takeuchi, Phys. Rev. Lett. 65, 1964 (1990).

[9] G. Altarelli and R. Barbieri, Phys. Lett. B 253, 161 (1990).

[10] See, e.g., J. Gunion and H. Haber, Nucl. Phys. B272, 1 (1986); M. Drees and K. Hagiwara, Phys. Rev. D 42, 1709 (1990).

[11] X. Li and E. Ma, “Tree-level Non-decoupling and the Super- 
symmetric Higgs Sector,' University of California at Riverside Report No. UCRHEP-T159, hep-ph/960838, 1996 (unpublished).

[12] C. D. Froggatt, R. G. Moorhouse, and I. G. Knowles, Phys. Rev. D 45, 2471 (1992); S. Bertolini, Nucl. Phys. B272, 77 (1986); W. Hollik, Z. Phys. C 32, 291 (1986); 37, 569 (1988).

[13] A. Longhitano, Phys. Rev. D 22, 1166 (1980); Nucl. Phys. B188, 118 (1981).

[14] R. Renken and M. Peskin, Nucl. Phys. B211, 93 (1983); T. Appelquist, et al., Phys. Lett. B 232, 211 (1989); A. Dobado and M. J. Herrero, ibid. 228, 495 (1989); J. F. Donoghue and C. Ramirez, ibid. 234, 88 (1990); B. Holdom and J. Terning ibid. 247, 88 (1990); A. Dobado, D. Espriu, and M. J. Herrero, ibid. 255, 405 (1991); A. Dobado, M. J. Herrero, and J. Terron, Z. Phys. C 50, 205 (1991); 50, 465 (1991); T. Appelquist and G. Triantaphyllou, Phys. Lett. B 278, 345 (1992); T. Appelquist and G.-H. Wu, Phys. Rev. D 48, 3235 (1993).
[15] D. Espriu and J. Matias, Phys. Lett. B 341, 332 (1995).

[16] J. F. Gunion, H. E. Haber, G. Kane, and S. Dawson, The Higgs Hunter's Guide (Addison-Wesley, Reading, MA, 1990), and references therein.

[17] Y. Grossman and Z. Ligeti, Phys. Lett. B 332, 373 (1994).

[18] M. Luscher and P. Weisz, Nucl. Phys. B290, 25 (1987).

[19] A. Pomarol and R. Vega, Nucl. Phys. B413, 3 (1994).

[20] B. Ovrut and J. Schnitzer, Phys. Rev. D 21, 3369 (1980); 22, 2518 (1980), and references therein.

[21] M. J. Herrero and E. Ruiz-Morales, Nucl. Phys. B418, 431 (1994); B437, 319 (1995).

[22] A. Nyffeler and A. Schenk, Ann. Phys. (N.Y.) 241, 301 (1995); Phys. Rev. D 53, 1494 (1996); S. Dittmaier and C. Grosse-Knetter, Nucl. Phys. B459, 497 (1996).

[23] G. Altarelli, R. Barbieri, and F. Caravaglios, Phys. Lett. B 349, 148 (1995), and references therein.

[24] G. Altarelli, Report No. hep-ph/9611239 (unpublished). 